\title{
Trabalho e agroindústria: olhares sobre doenças e riscos ocupacionais
}

\author{
Labour and agribusiness: perspectives on diseases and occupational hazards
}

Trabajo y agroindustria: perspectivas sobre enfermedades y riesgos laborales

Milena Nunes Alves de Sousa ${ }^{1,2 *}$, Patrícia Peixoto Custodio ${ }^{3,4}$, Antônio Wilson Júnior Ramalho Lacerda $^{4}$, Gildenia Pinto dos Santos Trigueiro ${ }^{1}$, Miguel Aguila Toledo', Vandezita Dantas de Medeiros Mazzaro', Ilana Andrade Santos do Egypto ${ }^{1}$, Tarciane Rosa de Vasconcelos Silva Barreto ${ }^{5}$, Jorge Vítor Barreto Araújo ${ }^{6}$, Flávia Regina Golçalves de Araújo ${ }^{7}$, Raquel Bezerra de Sá de Sousa Nogueira ${ }^{1}$, Everson Vagner de Lucena Santos ${ }^{1,2}$, Larissa de Araújo Batista Suárez ${ }^{2,8}$, Tiago Bezerra de Sá de Sousa Nogueira ${ }^{1}$, André Luiz Dantas Bezerra ${ }^{2}$.

\section{RESUMO}

Objetivo: Identificar, a partir de publicações nacionais e internacionais, os riscos ocupacionais e as doenças do trabalho na agroindústria. Método: Foi realizada Revisão Integrativa da Literatura, com buscas nos meses de abril e julho de 2019, na base de dados da Biblioteca Virtual em Saúde (BVS) e na Scientific Electronic Library Online (SCIELO), a partir da seguinte combinação entre os termos: Agroindústria AND "Riscos Ocupacionais" OR "risco ocupacional". Foram identificadas 452 publicações na BVS e 559 no SCIELO. Contudo, 27 constituíram a amostra final. Resultados: Foram identificados os seguintes riscos ocupacionais em ordem de importância: químicos, físicos, mecânicos, ergonômicos e biológicos. Quanto às doenças vinculadas ao trabalho no setor agroindustrial foram enfatizadas alcoolismo crônico e depressão. Conclusão: O setor possui relevância indiscutível, portanto, em decorrência dos riscos ocupacionais e dos agravos decorrentes da atividade é preciso repensar as estratégias em prol da saúde do trabalhador, com ações direcionadas à prevenção de doenças e a promoção da saúde no trabalho.

Palavras-Chave: Saúde do Trabalhador, Saúde, Doença, Exposição.

\begin{abstract}
Objective: To identify, from national and international publications, occupational risks and labor diseases in agribusiness. Method: An Integrative literature review was conducted, with searches in the months of April and July 2019, in the database of the Virtual Health Library (VHL) and the Scientific Electronic Library Online (SCIELO), from the following combination between the terms: agribusiness AND "Occupational hazards" OR "occupational hazard". 452 publications were identified in the VHL and 559 in SCIELO. However, 27 constituted the final sample. Results: The following occupational risks were identified in order of importance: chemical, physical, mechanical, ergonomic and biological. Regarding the diseases related to work in the agroindustrial sector, chronic alcoholism and depression were emphasized. Conclusion: The sector has undeniable relevance; therefore, due to the occupational risks and the problems resulting from the activity, it is necessary to rethink the strategies for the health of the worker, with actions directed to the prevention of diseases and Health promotion at work.
\end{abstract}

Key words: Occupational Health, Health, Disease, Exposure.

\footnotetext{
${ }^{1}$ Curso de Medicina do Centro Universitário de Patos - UNIFIP, Patos-PB, Brasil. *E-mail: minualsa@hotmail.com

${ }^{2}$ Curso de Enfermagem da Faculdade Vale do Pajeú, São José do Egito-PE, Brasil.

3Universidade Federal de Campina Grande, Cajazeiras-PB, Brasil.

${ }^{4}$ Universidade Federal de Campina Grande, Pombal-PB, Brasil.

${ }^{5}$ Curso de Medicina da Faculdade de Ciências Médicas da Paraíba, João Pessoa-PB, Brasil.

6 Universidade de Pernambuco, Recife-PE, Brasil.

${ }^{7}$ Faculdade de Medicina de Olinda, Olinda-PE, Brasil.

${ }^{8}$ Departamento de Psicologia da Universidade Católica de Pernambuco, Recife-PE, Brasil.
}

SUBMETIDO EM: $9 / 2019$

ACEITO EM: 9/2019

PUBLICADO EM: 10/2019 


\section{RESUMEN}

Objetivo: Identificar, con base en publicaciones nacionales e internacionales, los riesgos profesionales y enfermedades de trabajo en la agroindustria. Método: Se realizó revisión integradora de la literatura, con búsquedas en los meses de abril y julio de 2019, la base de datos de la Biblioteca Virtual en Salud (BVS) y Scientific Electronic Library Online (SCIELO), de la siguiente combinación de entre los términos: agroindustria AND "riesgos laborales" OR "riesgos laborales". Se identificaron 452 publicaciones en BVS y 559 en SCIELO. Sin embargo, 27 fueron la muestra final. Resultados: Se identificaron los riesgos ocupacionales siguientes en orden de importancia: químicos, físicos, ergonómicos, mecánicos y biológicos. En cuanto a enfermedades relacionadas con el sector agroindustrial fueron alcoholismo crónico y la depresión. Conclusión: el sector tiene importancia innegable, por lo tanto, como consecuencia de riesgos profesionales y enfermedades derivadas de la actividad es necesario replantear las estrategias para la salud del trabajador, con acciones dirigidas a la prevención de la enfermedad y la promoción de la salud del lugar de trabajo.

Palabras clave: Salud de los Trabajadores, Salud, Enfermedad, Exposición.

\section{INTRODUÇÃO}

Segundo o SENAR-AR/PE (2013), pode-se entender juridicamente agroindústria como "o produtor rural pessoa jurídica cuja atividade econômica seja a industrialização de produção própria ou de produção própria e adquirida de terceiros". Ainda, "desenvolve duas atividades em um mesmo empreendimento econômico, com departamentos, divisões ou setores rural e industrial distintos".

Atualmente, a agroindústria se destaca no setor do agronegócio, com sinais de amadurecimento tecnológico e gerencial dos produtores, em um conjunto de atividades relacionadas à transformação da matéria prima advinda da agricultura, pecuária, silvicultura, aquicultura e pesca. De acordo com o objetivo da agroindústria, o grau de transformação é variável (VIEIRA FILHO JER e VIEIRA ACP, 2013). Envolve várias etapas produtivas, entre os quais se citam: processamento, insumos, distribuição e produção primária (OLISZESKI CAN, 2011).

No Brasil, os grandes complexos agroindustriais estão concentrados na indústria de alimentos, bebidas, papel, celulose e tecelagem (SOUSA MNA, MEDEIROS AC e MARACAJÁ PB, 2018). É responsável por 25\% do Produto Interno Bruto (PIB) do país, gerando muitos empregos diretos e indiretos, abarca35\% da mão-deobra brasileira (WILKINSON J, 2009).

Apesar dos resultados positivos citados, a atividade agroindustrial também pode ter repercussões negativas sobre a saúde dos trabalhadores. De modo particular, em indústria pecuária, estudo no setor avícola do Paraná indicou, entre os anos de 2006-2008, aproximadamente 660 benefícios previdenciários decorrentes de agravos de ordem mental e comportamental, bem como osteomioarticular, com média anual de 220 (HECK; THOMAZ JÚNIOR A, 2012).

Para averiguação do processo de trabalho é possível refletir sobre duas vertentes, conhecidas pelas cargas/riscos e desgastes. As primeiras podem ser subclassificadas também em dois grupos 1) externas ao corpo do trabalhador - cargas químicas, físicas, mecânicas e biológicas ou 2) as que só são materializáveis na corporeidade humana, conhecidas como psíquicas e fisiológicas, estas relacionam-se a posturas, esforços repetitivos e outros, as psíquicas podem provocar tensões prolongadas ou sobrecargas psicológicas, a exemplo da atenção constante, cobrança, ritmo e supervisão constante. As cargas físicas conduzem a acidentes e, especialmente, problemas biológicos. É notável, portanto, que estes fatores atuam no processo de trabalho e podem afetar a saúde do trabalhador (PINTO NF, MUROFUSE NT e CARVALHO M, 2015).

Afirma-se que estas cargas e desgastes são potencialmente prejudiciais ao trabalhador agroindustrial. Assim sendo, esse estudo tem como objetivo identificar, a partir de publicações nacionais e internacionais, os riscos ocupacionais e as doenças do trabalho na agroindústria. Justifica-se por tentar contemplar uma temática ainda pouco abordada, a fim de tentar suprir os hiatos existentes, uma vez que os estudos no setor agroindustrial têm basicamente contemplado as questões inerentes à gestão e produtividade. É fundamental que aos trabalhadores envolvidos na agroindústria sejam repassadas informações sobre sua saúde e 
segurança, "pois a percepção e o conhecimento de risco são as premissas básicas para que o indivíduo ocupacionalmente exposto possa efetivamente se proteger" (SANTOS MBG, et al., 2016, p. 2). Ante as ponderações, propôs-se identificar, a partir de publicações nacionais e internacionais, os riscos ocupacionais e as doenças do trabalho na agroindústria.

\section{METODOLOGIA}

Trata-se de uma Revisão Integrativa Literária, é composta de cinco etapas: formulação do problema; coleta de dados; avaliação dos dados; análise e interpretação dos dados e divulgação dos dados.

Inicialmente elencou-se uma temática relacionada à relação trabalho, saúde e adoecimento. Portanto, a questão de pesquisa primária indagou <<quais os riscos ocupacionais e as doenças do trabalho na agroindústria?>>. Definido o questionamento, precedeu-se a determinação dos termos de busca. Foi feita a seguinte combinação das palavras-chaves em português: Agroindústria $A N D$ "Riscos Ocupacionais" OR "Risco Ocupacional".

Posteriormente, realizou-se a busca na literatura nos bancos de dados da Biblioteca Virtual em Saúde (BVS) e na Biblioteca Virtual da Scientific Electronic Library Online (SCIELO) no mês de abril a agosto de 2019. Foram identificadas 463 publicações na BVS e 583 no SCIELO.

O material foi selecionado a partir dos filtros de inclusão: tempo (2015-2019), descritores no título e artigo como formato de documento. Excluíram-se documentos que não estavam no formato de artigo ou as repetições, permanecendo ao menos uma vez. Assim, a amostragem foi de 27 documentos (Figura 1).

Figura 1 - Estudos selecionados segundo a base de dados.

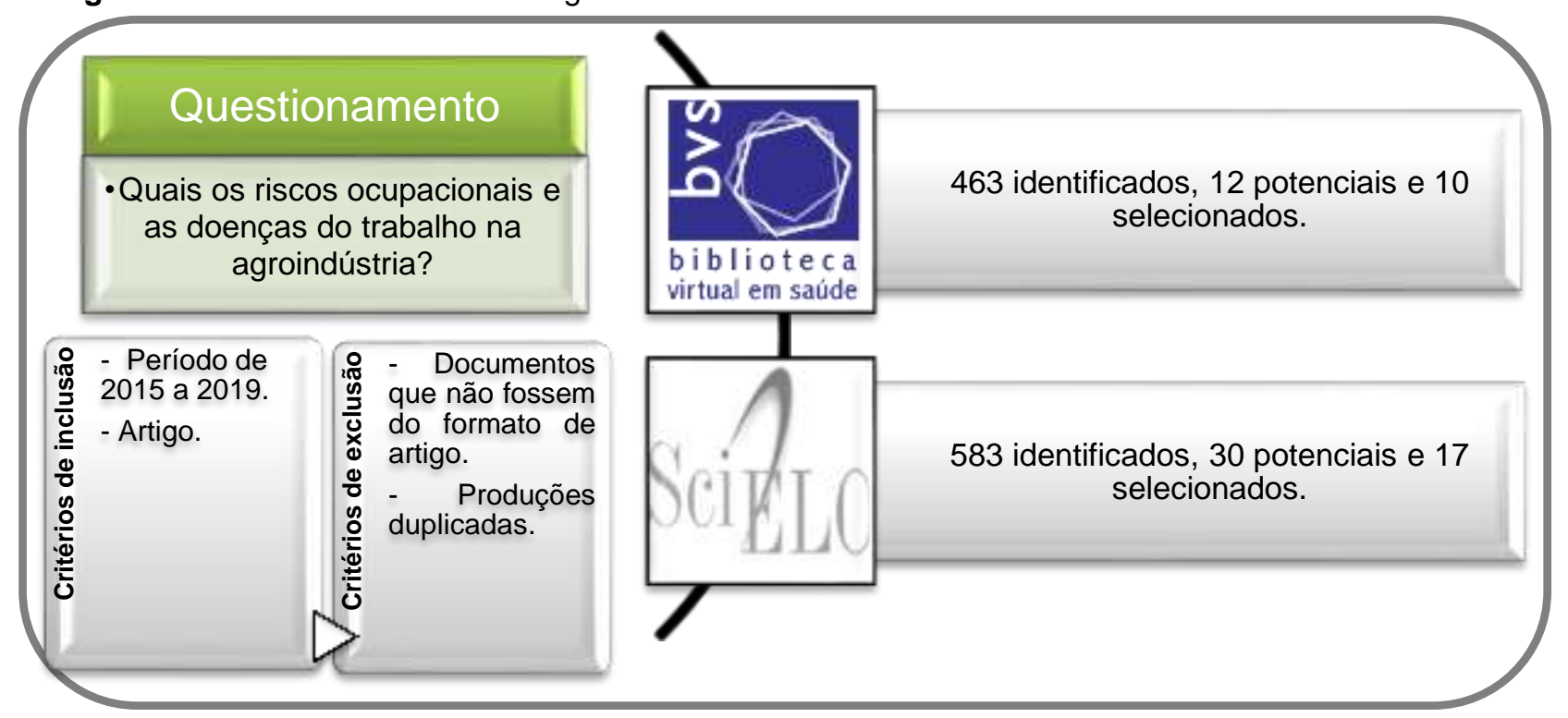

Fonte: Sousa MNA, et al., 2019.

De posse das publicações selecionadas, as mesmas foram lidas e categorizadas em: 1) Riscos Ocupacionais e 2) Doenças Relacionadas ao Trabalho. Para compor a matriz de síntese, extraíram-se as seguintes informações dos artigos: Autor(es)/Ano, Título, Base de Dados; Idioma; Periódico. Os achados foram analisados, discutidos e a revisão concluída com análise, interpretação e divulgação dos dados.

\section{RESULTADOS}

Dos 27 documentos selecionados nesta revisão, 63\% ( $n=18)$ foram publicados no SCIELO, 67\% ( $n=18)$ em idioma internacional e 41\% ( $n=11)$ no ano de 2016 (Quadro 1). 
Quadro 1 - Caracterização quanto aos autores, ano, título, base de dados, idioma, periódico.

\begin{tabular}{|c|c|c|c|c|}
\hline \multicolumn{5}{|c|}{ BVS } \\
\hline Número & Autor(es)/Ano & Título & Idioma & Periódico \\
\hline N1 & $\begin{array}{l}\text { Gómez CJR e Espinosa HR } \\
\text { (2017) }\end{array}$ & $\begin{array}{l}\text { Influencia de los recursos y las dinámicas del territorio en el sostenimiento } \\
\text { de la agroindustria rural panelera de supía, caldas }\end{array}$ & Internacional & Rev. Luna Azul \\
\hline N2 & Azevedo JC, et al. (2017) & Fruit agribusiness waste as an additive in elephant grass silage & Internacional & $\begin{array}{l}\text { Semina: Ciências } \\
\text { Agrárias }\end{array}$ \\
\hline N3 & $\begin{array}{l}\text { Machado LF, Murofuse NT e } \\
\text { Martins JT (2016) }\end{array}$ & $\begin{array}{l}\text { Vivências de ser trabalhador na agroindústria avícola dos usuários da } \\
\text { atenção à saúde mental }\end{array}$ & Nacional & Saúde debate \\
\hline N4 & Penha EDM, et al. (2016) & $\begin{array}{l}\text { Aproveitamento de resíduos da agroindústria do óleo de dendê para a } \\
\text { produção de lipase por Aspergillus Níger }\end{array}$ & Nacional & Cienc. Rural \\
\hline N5 & Aranda P e Vásquez MCC (2016) & $\begin{array}{l}\text { El campo de la agroindustria en el noroeste de México y la salud de sus } \\
\text { jornaleras: una propuesta de estúdio }\end{array}$ & Internacional & $\begin{array}{l}\text { Universidad Nacional } \\
\text { de Lanús }\end{array}$ \\
\hline N6 & Ferreira $\mathrm{ACH}$, et al. (2016) & $\begin{array}{l}\text { In situ degradability of elephant grass ensiled with increasing levels of } \\
\text { pineapple agro-industrial byproduct }\end{array}$ & Internacional & $\begin{array}{l}\text { Semina: Ciências } \\
\text { Agrárias }\end{array}$ \\
\hline N7 & Braga AP, et al. (2016) & Ruminal degradability of agro-industrial fruit residues & Internacional & $\begin{array}{l}\text { Semina: Ciências } \\
\text { Agrárias }\end{array}$ \\
\hline N8 & Volpato RM, et al. (2015) & Coprodutos da agroindústria na alimentação de leitões & Nacional & Cienc. Rural \\
\hline N9 & Almeida JCS, et al. (2015) & $\begin{array}{l}\text { Desempenho, medidas corporais, rendimentos de carcaça e cortes, e } \\
\text { qualidade de carne em cordeiros alimentados com resíduos da } \\
\text { agroindústria processadora de frutas }\end{array}$ & Nacional & $\begin{array}{l}\text { Semina: Ciências } \\
\text { Agrárias }\end{array}$ \\
\hline N10 & Maia ISAS, et al. (2015) & $\begin{array}{l}\text { Valor Nutritivo de Silagens de Capim Elefante com níveis crescentes de } \\
\text { Resíduo da Agroindústria da Acerola }\end{array}$ & Nacional & $\begin{array}{l}\text { Acta Veterinaria } \\
\text { Brasilica }\end{array}$ \\
\hline \multicolumn{5}{|c|}{ SCIELO } \\
\hline N11 & $\begin{array}{l}\text { Alarcón S, Marcucci D e Quiroga } \\
\text { M (2018) }\end{array}$ & Territorialidad campesina y agroindustria en el río Cimitarra & Internacional & $\begin{array}{l}\text { Bitácora Urbano } \\
\text { Territorial }\end{array}$ \\
\hline $\mathrm{N} 12$ & Martínez AC (2018) & $\begin{array}{l}\text { Ciertos espacios de ocupación La relación agroindustria-protestantismo en } \\
\text { la formación de una región fronteriza entre México y Estados Unidos }\end{array}$ & Internacional & Estudios Fronterizos \\
\hline $\mathrm{N} 13$ & Deamici KM, et al. (2018) & Development of cookies from agroindustrial by-products & Internacional & Rev. Bras. Frutic. \\
\hline
\end{tabular}

REAS/EJCH | Vol.Sup.33 | e1570 | DOI: https://doi.org/10.25248/reas.e1570.2019 Página 4 de 11 


\begin{tabular}{|c|c|c|c|c|}
\hline N14 & $\begin{array}{l}\text { Zavala P, Macario G e Vichi FF } \\
(2018)\end{array}$ & $\begin{array}{l}\text { El sector citrícola de Nuevo León: caracterización del sistema } \\
\text { agroalimentario como plataforma de integración del productor con la } \\
\text { agroindústria }\end{array}$ & Internacional & Región y sociedade \\
\hline N15 & $\begin{array}{l}\text { Huerta-Dueñas M e Sandoval- } \\
\text { Godoy SA (2018) }\end{array}$ & $\begin{array}{l}\text { Sistemas de calidad como estrategia de ventaja competitiva en la } \\
\text { agroindustria alimentaria }\end{array}$ & Internacional & $\begin{array}{l}\text { Agricultura, sociedac } \\
\text { y desarrollo }\end{array}$ \\
\hline N16 & Olivo PM, et al. (2017) & $\begin{array}{l}\text { Assessing the nutritional value of agroindustrial co-products and feed } \\
\text { through chemical composition, in vitro digestibility, and gas production } \\
\text { technique }\end{array}$ & Internacional & $\begin{array}{l}\text { Acta Scientiarum. } \\
\text { Animal Sciences }\end{array}$ \\
\hline N17 & Souza HAD, et al. (2017) & $\begin{array}{l}\text { Quantificação de nutrientes nos frutos de goiabeiras adubadas com } \\
\text { subproduto da agroindústria processadora de goiabas }\end{array}$ & Nacional & $\begin{array}{l}\text { Rev. de Ciências } \\
\text { Agrárias }\end{array}$ \\
\hline N18 & $\begin{array}{l}\text { Murcia-Pardo ML e Ramírez- } \\
\text { Durán J (2017) }\end{array}$ & $\begin{array}{l}\text { Reconversión del sistema regional de producción de semilla de caña para } \\
\text { la agroindustria panelera en Boyacá y Santander }\end{array}$ & Internacional & $\begin{array}{l}\text { Ciencia y Tecnología } \\
\text { Agropecuaria }\end{array}$ \\
\hline N19 & Sehnem S e Oliveira GPD (2016) & $\begin{array}{l}\text { Green Supply Chain Management: an Analysis of the Supplier-Agro } \\
\text { Industry Relationship of a Southern Brazilian Company }\end{array}$ & Internacional & BBR, Braz. Bus \\
\hline N20 & $\begin{array}{l}\text { Ocón HBF, Guzmán GO e } \\
\text { Ocampo GSV (2016) }\end{array}$ & $\begin{array}{l}\text { Dinamismo de la agroindustria en el Corredor Costero de Chiapas, } \\
\text { México. Coordinación y competitividad territorial }\end{array}$ & Internacional & Mundo agrário \\
\hline N21 & Francisco AP, et al. (2016) & $\begin{array}{l}\text { Ozônio troposférico e compostos orgânicos voláteis em região impactada } \\
\text { pela agroindústria canavieira }\end{array}$ & Nacional & Quím. Nova \\
\hline N22 & $\begin{array}{l}\text { Pérez-Cruz JR e Rappo-Miguez } \\
\text { SE (2016) }\end{array}$ & $\begin{array}{l}\text { Opciones de política ambiental para garantizar la sustentabilidad de la } \\
\text { agroindustria azucarera en Puebla, México }\end{array}$ & Internacional & $\begin{array}{l}\text { Agricultura, sociedac } \\
\text { y desarrollo }\end{array}$ \\
\hline N23 & Mesquita FC e Furtado AT (2016) & $\begin{array}{l}\text { Expansão da agroindústria canavieira e qualificação da mão-de-obra em } \\
\text { Goiás (2006-2013) }\end{array}$ & Nacional & $\begin{array}{l}\text { Sociedade \& } \\
\text { Natureza }\end{array}$ \\
\hline N24 & Silva RB, et al. (2015) & $\begin{array}{l}\text { Cinzas de biomassa geradas na agroindústria do cacau: caracterização e } \\
\text { uso em substituição ao cimento }\end{array}$ & Nacional & Ambiente Construídc \\
\hline N25 & $\begin{array}{l}\text { Aguilar-Rivera N, Alejandre- } \\
\text { Rosas J e Espinosa-López R } \\
\text { (2015) }\end{array}$ & $\begin{array}{l}\text { Evaluación emergy y LCA en la agroindustria azucarera de Veracruz, } \\
\text { México }\end{array}$ & Internacional & Cultivos Tropicales \\
\hline N26 & Gurgel MN, et al. (2015) & $\begin{array}{l}\text { Technology for sugarcane agroindustry waste reuse as granulated } \\
\text { organomineral fertilizer }\end{array}$ & Internacional & Eng. Agríc \\
\hline N27 & Calvache OLB, et al. (2015) & $\begin{array}{l}\text { Ácidos grasos del hongo funcional pleurotus ostreatus cultivado en } \\
\text { residuos sólidos agroindustriales }\end{array}$ & Internacional & Producción + Limpia \\
\hline
\end{tabular}

REAS/EJCH | Vol.Sup.33 | e1570 | DOI: https://doi.org/10.25248/reas.e1570.2019 Página 5 de 11 
De acordo com as categorias, 96,4\% $(n=27)$ deles se enquadram na categoria Riscos Ocupacionais e $3,6 \%(n=1)$ na de Doenças Relacionadas ao Trabalho. Este último corresponde à identificação N3 da tabela anterior.

Na categoria 1 ainda foi possível identificar os tipos de riscos no trabalho da agroindústria de acordo com sua classificação, em que o Risco Químico foi o mais citado (28,4\%; $n=17)$ (Figura 2). Ademais, os riscos químicos, físicos e mecânicos foram os mais citados $(75,1 \%)$ da amostra.

Figura 2 - Categorização dos estudos selecionados.

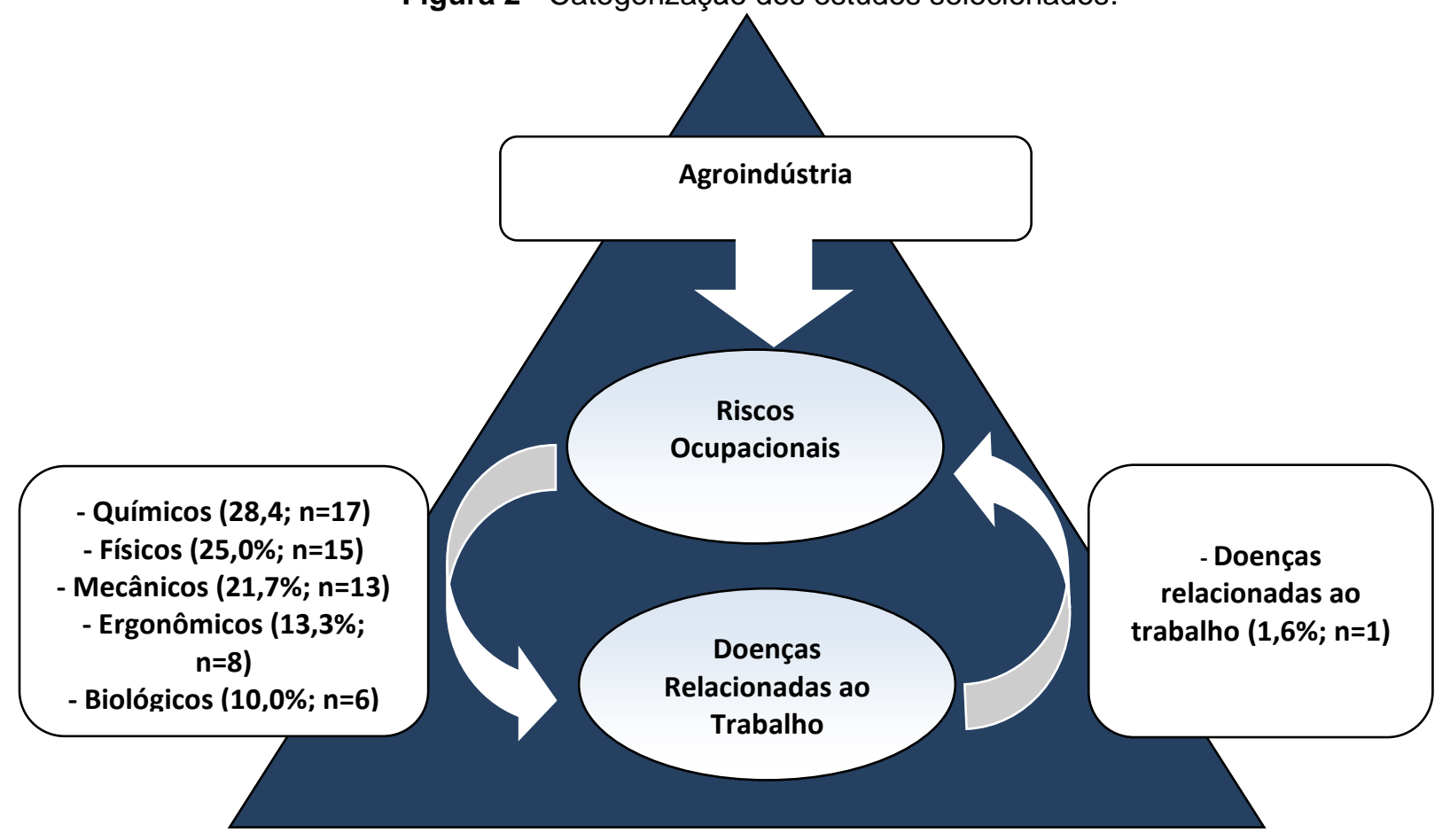

Fonte: Sousa MNA, et al., 2019.

\section{DISCUSSÃO}

Os estudos selecionados nesta Revisão Integrativa da Literatura contemplaram duas categorias: 1) Riscos Ocupacionais e 2) Doenças Relacionadas ao Trabalho no setor agroindustrial, a partir de publicações nacionais e internacionais, uma vez que as condições de trabalho na agroindústria caracterizam-se pela exposição a inúmeros fatores que prejudicam a saúde do trabalhador.

O trabalhador, enquanto detentor da força de trabalho, pode vivenciar situações de conflitos decorrentes de seu processo laboral. De um lado, a certeza de garantida de seu sustento e de sua família, de outro, as intercorrências sobre sua saúde e risco de morte (MACHADO LF, MUROFUSE NT e MARTINS JT, 2016). Assim sendo, considerando os riscos ocupacionais, todos os estudos destacaram em menor ou maior grau a sua presença em decorrência do processo de trabalho na agroindústria. De modo específico, foi possível verificar os seguintes achados:

- Químicos - manejo com borra alcalina, com ácido sulfúrico e detergentes específicos, nitrogênio amoniacal, Exposição ao Monóxido de Carbono e a outras substâncias tóxicas, contato com agrotóxicos, produção e contato com gás (PENHA EDM, et al., 2016; ZAVALA P, MACARIO G e VICHI FF, 2018).

A borra alcalina, subproduto do refino de óleos comestíveis, é originada de uma mistura de sabão, óleo arrastado, substâncias insaponificáveis, gomas, fosfatídeos, pigmentos, água e ácidos graxos (MURUCI LNM, et al., 2012).

O ácido sulfúrico é um composto químico, sendo classificado como um ácido mineral forte. O mesmo deve ser manuseado com cuidado, devido ele ser corrosivo para os tecidos do organismo, a inalação dos vapores 
pode causar graves danos aos pulmões, o contato com a pele ou olhos pode resultar queimaduras e cegueira (TEVES MLU, 2003).

No processo de digestão anaeróbia, as proteínas encontradas no substrato são degradadas e convertidas em nitrogênio amoniacal, o qual pode encontrar-se sob as formas íon amônio. Essa é uma fonte essencial de nitrogênio para os micro-organismos envolvidos no processo anaeróbio, no entanto, quando presente em altas concentrações, a amônia pode representar toxicidade aos micro-organismos (SHENG K, et al., 2013).

O Monóxido de Carbono é fabricado quando gás, madeira, carvão ou demais combustíveis são queimados. Muitas vezes, acumula-se quando aquecedores e aparelhos de cozimento a gás falham ou têm ventilação inadequada, o que pode causar envenenamento (WOOD RND, 2018).

Os agrotóxicos são amplamente utilizados no meio rural e sua vasta utilização desprovida de medidas de biossegurança apropriadas, vem provocando impactos potenciais tanto para o ambiente quanto para a saúde dos trabalhadores. A intoxicação por essas substâncias químicas está aumentando de forma acelerada, principalmente dos trabalhadores rurais através de exposição ocupacional (RIBEIRO DS e PEREIRA TS, 2016).

- Físicos - manejo com altas e baixas temperaturas $\left(0^{\circ}-121^{\circ} \mathrm{C}\right)$, ambiente com ruídos, trabalho na presença de umidade no setor (85\%) (PÉREZ-CRUZ JR e RAPPO-MIGUEZ SE, 2016; AZEVEDO JC, et al., 2017).

$O$ armazenamento em câmaras frias conserva os alimentos frescos em temperaturas baixas e essa ação atrasa o desenvolvimento de microrganismos e a atividade das enzimas (SOARES R, 2010). Diante de circunstâncias térmicas extremas, como temperaturas acima dos $40^{\circ} \mathrm{C}$ o corpo apresenta reflexos de desequilíbrio térmico, podendo chegar á Óbito. Assim havendo indisposição causada por altas temperaturas, é ideal parar o trabalho e aguardar o equilíbrio do corpo, evitando agravos á saúde do trabalhador ou mesmo acidentes de trabalho (SILVA TL e ALMEIDA VC, 2010).

Sobre a presença de umidade no setor, é permitido dizer que a velocidade do ar, além da umidade relativa do ar e da temperatura, em um ambiente de trabalho, interfere no equilíbrio térmico (MARRA GC, et al., 2017).

A exposição habitual às grandes alterações de temperaturas eleva a incidência de gripes, amidalites, laringites, bronquites, broncopneumonias, pneumonias e rinites (JOHNSON ES, et al., 2011).

Por fim, em alta intensidade e com exposição continuada, o ruído pode causar alterações estruturais na orelha interna, que determinam a ocorrência da Perda Auditiva Induzida por Ruído (PAIR), e é o motivo de agravo mais frequente à saúde dos trabalhadores (BARCELOS DD e ATAIDE SG, 2014).

O trabalho com exposição à umidade é reconhecido como aquele que envolve a frequente imersão das mãos em água, a lavagem frequente ou intensa das mãos ou o uso frequente de luvas impermeáveis. Os profissionais submetidos a tais exposições são acometidos de eczema e dermatite de contato nas mãos (ZANI MLC, LAZZARINI R e SILVA-JUNIOR JS, 2017).

- Mecânicos - manejo com instrumentos perfurantes e cortantes, com grandes máquinas de corte (moinho de facas, triturador) e selagem, manuseio de máquinas de prensa e falta de Equipamento de Proteção Individual (EPI) (MACHADO LF, MUROFUSE NT e MARTINS JT, 2016; HUERTA-DUEÑAS M e SANDOVALGODOY SA, 2018). Dentre as atividades agrícolas, as atividades mecanizadas são as que oferecem maiores riscos de acidentes. Essas práticas pressupõem não somente o emprego de máquinas, mas a interferência do homem, formando um sistema homem-máquina. Este atributo necessita de medidas para prevenção de acidentes. Quando as ações técnicas coletivas e as medidas administrativas não são suficientes para reduzir a exposição a um nível aceitável, deverá fornecer-se aos trabalhadores um equipamento de proteção individual (EPI) apropriado. Desta forma o equipamento de proteção individual é todo instrumento de uso individual, destinado à defesa a saúde e a integridade física do operário (CEARÁ, 2011).

- Ergonômicos - intensa jornada de trabalho, múltiplas atividades setoriais, baixos salários, assédio sexual, discriminação sexual, mão de obra escrava, postura incorreta (GÓMEZ CJR e ESPINOSA HR, 2017; MARTÍNEZ AC, 2018). 
Em função das dificuldades desses contextos laborais sobre intensa jornada de trabalho bem como do reconhecimento dos agravos à saúde dos trabalhadores decorrentes, vêm sendo adotadas ações para melhorarem as condições de trabalho neste setor de produção (MAGRO MLPD, et al.,2014).

A pressão salarial para baixo do trabalho assalariado na agricultura justifica-se, segundo a razão empresarial, a partir de critérios como nível de escolaridade e qualificação profissional (ARAUJO LB, 2018).

Estudo possibilitou a compreensão do assédio sexual no trabalho a partir de quatro perspectivas: assédio sexual no trabalho como tipo de violência psicológica no trabalho; assédio sexual no trabalho compreendido a partir de relações de poder; assédio sexual no trabalho compreendido a partir da desigualdade de gênero; e assédio sexual no trabalho compreendido a partir do aspecto jurídico (FONSECA TS, et al., 2018).

- Biológicos - manuseio com resíduos agroindustriais (banana, maracujá, manga, abacaxi, caju, goiaba, tamarindo, acerola, batata palha, pipoca doce), manuseio com fezes e urina animal (BRAGA AP, et al., 2016; e FERREIRA ACH, et al., 2016).

A agroindústria pode ser definida como o conjunto de atividades referente à transformação de matériasprimas provenientes da agricultura, pecuária, aquicultura ou silvicultura em produtos. Logo uma problemática relacionada à agroindústria brasileira é a grande concentração de resíduos originado durante 0 processamento/beneficiamentos de suas matérias primas. Na maioria dos casos, os resíduos proveniente das agroindústrias, não são tratados e apresentam uma disposição ambientalmente inadequada, com potenciais ricos de contaminação dos solos e águas (VIANA LG e CRUZ OS, 2016).

Sobre os riscos citados mais frequentes neste estudo foi possível evidenciar como químico o uso de trabalho em laboratório, com utilização de métodos para garantir um resultado final seguro, no entanto, tais métodos muitas vezes deixam os trabalhadores do setor expostos a substâncias compostas ou produtos químicos. Para o risco físico foi identificado o ruído do ambiente de trabalho devido o funcionamento das máquinas de diferentes portes, soma-se contato com altas e baixas temperaturas são bem citadas devido ao uso de estufa, forno e câmara fria para manter a qualidade desejada dos produtos. Por fim, relacionado aos riscos mecânicos apontam-se a utilização de ferramentas como facas, serras e iluminação inapropriadas no setor, risco de choque elétrico devido ao mau funcionamento ou encapes de fios.

O trabalho com exposição a substâncias químicas pode levar casos de mortes, dermatoses de pele ou desenvolvimento de câncer (CARVALHO LVB, et al., 2017). Concomitantemente, os ruídos intensos tendem a prejudicar tarefas que exigem atenção, concentração mental, ou repetitividade de movimentos, além de provocarem perturbações do estado de atenção, descanso e aumento do estresse.

Apesar de serem muitos os riscos na atividade agrícola, os principais entraves desta exposição centramse na falta de gestão de riscos entre os trabalhadores do setor e, também, algumas características pessoais dos agricultores (SOUSA MNA, ASSIS EV e FEITOZA ANA, 2014). Características individuais parecem, ainda, contribuir para que a exposição aos riscos sejam mais evidentes. No setor, há uma "prevalência dos acidentes entre os homens, ocorrendo predominantemente os acidentes típicos, as doenças ocupacionais e acidentes de trajeto" (JESUS CS e BRITO TA, 2009, p. 141).

Reforça-se que o setor agroindustrial possui relevância indiscutível e em decorrência das repercussões do trabalho na saúde do trabalhador da área devido aos riscos ocupacionais, é preciso repensar as estratégias em prol da saúde do grupo, com ações direcionadas à prevenção de doenças e a promoção da saúde no trabalho.

As doenças ocupacionais são aquelas provocadas pelo exercício da atividade ou em função de circunstâncias especiais de trabalho. Os servidores e empregadores precisam ficar atentos em relação os principais motivos de doenças ocupacionais e a como evitá-las, buscando o constante aprimoramento das condições de saúde e segurança do ambiente de trabalho. Além disso, é preciso estar atento aos primeiros sinais de desconforto físico ou mental (SOUSA MNA, ASSIS EV e FEITOZA ANA, 2014)

Dentre os agravos citados neste estudo tem-se o alcoolismo crônico e depressão, citados na pesquisa de Machado LF, Murofuse NT e Martins JT (2016) (N3). O alcoolismo é a dependência do indivíduo ao álcool, 
considerada doença pela Organização Mundial da Saúde. O uso constante, descontrolado e progressivo de bebidas alcoólicas pode comprometer seriamente o bom funcionamento do organismo, levando a consequências irreversíveis (BRASIL, 2015). Neste estudo esta doença foi relatada por trabalhadores que se sentiam insatisfeitos pelas condições de vida, salários e da carga horária de trabalho excessiva.

A prevalência de consumo abusivo de álcool entre os canavieiros é intensa e os principais fatores associados ao consumo são a insatisfação com o trabalho, o sentimento de tristeza ao final da jornada de trabalho e a baixa qualidade de vida (BECK FILHO JAB, AMORIM AM e MAIA HF, 2016).

Por sua vez a Organização Mundial da Saúde indica o consumo de álcool como um dos graves problemas de Saúde Pública na atualidade. Estima-se que existam cerca de dois bilhões de indivíduos em todo o mundo que ingerem bebidas alcoólicas e que 76,3 milhões que fazem uso de álcool com diagnóstico de transtorno mental (dependência) (BRASIL, 2015).

Quanto à depressão, as causas estão associadas a baixos salários, assédio sexual, discriminação sexual, intolerância religiosa, discriminação racial. Os funcionários afetados relataram ter acompanhamento pelo Centro de Atenção Psicossocial (CAPS) local.

A depressão consiste num transtorno mental que recebe atenção e estudos de especialistas há várias décadas. Assim, é possível dizer que o trabalho desenvolvido nas agroindústrias é capaz de corroborar para o aumento da depressão entre os trabalhadores do setor, sendo considerada como acidente do trabalho (GOLDSCHMIDT R e FERRAZ D, 2012).

A partir dos agravos outrora listados e aos militantes do campo da Saúde do Trabalhador ressalta-se que o debate sobre o tema ainda é bastante atual e instigador.

\section{CONSIDERAÇÕES FINAIS}

A partir da síntese desta revisão integrativa e mediante discussão dos dados, concebe-se que o presente estudo atingiu o objetivo proposto, pois desvendou achados fundamentais quanto aos riscos inerentes às atividades na agroindústria e seu poder de comprometimento da saúde do trabalhador do setor. Destaques foram ilustrados aos riscos químicos, físicos e mecânicos, embora também tenham se identificado os ergonômicos e biológicos. Ademais, e com igual importância, deve-se reforçar e alertar para as doenças do trabalho no setor identificadas, em que se sobressaíram o alcoolismo crônico e depressão.

\section{REFERÊNCIAS}

1. ARAUJO LB. A política salarial para o trabalho na agricultura no complexo agroindustrial canavieiro da paraíba. Anais. 16ํㅡㄹ ENCONTRO NACIONAL DE PESQUISADORES EM SERVIÇO SOCIAL, 02 a 07 de dezembro de 2018. Vitória-ES; 2018. Disponível em: <www.periodicos.ufes.br>. Acesso em: 22 ago 2019.

2. AZEVEDO JC, et al. Fruit agribusiness waste as an additive in elephant grass silage. Semina: Ciências Agrárias,2017; 38(4):1987-1999.

3. BARCELOS DD, ATAIDE SG. Análise do risco ruído em indústria de confecção de roupa. Rev. CEFAC, 2014; 16(1);3949.

4. BECK FILHO JAB, AMORIM AM, MAIA HF. Consumo de Álcool entre os Trabalhadores do corte da Cana-de-Açúcar. Revista Pesquisa em Fisioterapia, 2016;6(3):306-316.

5. BRAGA AP, et al. Ruminal degradability of agro-industrial fruit residues. Semina: Ciências Agrárias, 2016;37(1):279292.

6. BRASIL. Ministério da Saúde. Alcoolismo. 2015. Disponível em:<http://bvsms.saude.gov.br/dicas-em-saude/412alcoolismo>. Acesso em: 21 ago 2019.

7. CARVALHO LVB, et al. Exposição ocupacional a substâncias químicas, fatores socioeconômicos e Saúde do Trabalhador: uma visão integrada. Saúde Debate, 2017;41(esp.):313-326.

8. CEARÁ. Secretaria de educação. Agroindústria - Saúde, Segurança e Ergonomia no Trabalho. 2011.

9. GOLDSCHMIDT R, FERRAZ D. Depressão acometida ao trabalhador agroindustriário: restrição à eficácia do direito fundamental social ao trabalho digno. Revista de Direitos e Garantias Fundamentais (FDV), 2012;12:327-358. 
10. FERREIRA ACH, et al. In situ degradability of elephant grass ensiled with increasing levels of pineapple agro-industrial byproduct. Semina: Ciências Agrárias, 2016;37(4):2807-2818.

11. FONSECA TS, et al. Assédio Sexual no Trabalho: Uma Revisão Sistemática de Literatura. Ciencias Psicológicas,2018;12(1):25-34.

12. GÓMEZ CJR e ESPINOSA HR. Influencia de los recursos y las dinámicas del territorio en el sostenimiento de la agroindustria rural panelera de Supía, Caldas. Revista Luna Azul, 2017;44:288-210.

13. HUERTA-DUEÑAS M, SANDOVAL-GODOY SA. Sistemas de calidad como estrategia de ventaja competitiva en la agroindustria alimentaria. Agricultura, sociedad y desarrollo,2018;15(1);19-28.

14. JESUS CS e BRITO TA. Estudo dos acidentes de trabalho no meio rural: análise dos processos e condições de trabalho. Rev.Saúde.Com 2009; 5(2): 141-146.

15. JOHNSON ES, et al. Mortalidade em trabalhadores empregados em matadouros de suínos e plantas de processamento. Res. Nova lorque, 2011;111(6):871-876.

16. MACHADO LF, MUROFUSE NT, MARTINS JT. Vivências de ser trabalhador na agroindústria avícola dos usuários da atenção à saúde mental. Saúde Debate, 2016;40(110):134-147.

17. MAGRO MLPD, et al. Intensificação e prolongamento da jornada de trabalho nas indústrias de abate e processamento de carnes e seus impactos na saúde dos trabalhadores. Cadernos de Psicologia Social do Trabalho, 2014;17(1):67-83.

18. MARRA GC, et al. O. Avaliação dos riscos ambientais na sala de abate de um matadouro de bovinos. Saúde Debate, 2017;41(esp):175-187.

19. MARTÍNEZ AC. Ciertos espacios de ocupación La relación agroindustria-protestantismo en la formación de una región fronteriza entre México y Estados Unidos. Estudios fronterizos, 2018;19:1-20.

20. MURUCI LNM, et al. Produção de lipase de Aspergillus niger utilizando co-produtos da indústria de refino de óleos. In: Anais... CONGRESSO BRASILEIRO DE ENGENHARIA QUÍMICA,19., 2012, Búzios, RJ. 2012. Disponível em: <https://www.alice.cnptia.embrapa.br/bitstream/doc/934730/1/2012108.pdf >. Acesso em: 15 ago 2019.

21. OCÓN HBF, GUZMÁN GO, OCAMPO GSV. Dinamismo de la agroindustria en el Corredor Costero de hiapas, México. Coordinación y competitividad territorial. Mundo agrario,2016:7(36):1-20.

22. OLISZESKI CAN. Modelos de planejamento agrícola: um cenário para otimização de processos agroindustriais. 2011. 99 f. Dissertação [Mestrado em Engenharia de Produção] - Universidade Tecnológica Federal do Paraná; 2010.

23. PENHA EDM, et al. (2016). Aproveitamento de resíduos da agroindústria do óleo de dendê para a produção de lipase por Aspergillus niger. Ciência rural, 2016;46(4):755-761.

24. PÉREZ-CRUZ JR, RAPPO-MIGUEZ SE. Opciones de política ambiental para garantizar la sustentabilidad de la agroindustria azucarera en Puebla, México. Agricultura, sociedad y desarrollo, 2016;13(2):193-216.

25. PINTO NF, MUROFUSE NT, CARVALHO M. Processo e cargas de trabalho e a saúde dos trabalhadores na sericicultura: uma revisão. Revista Brasileira de Saúde Ocupacional, 2015;40(132):237-247.

26. RIBEIRO DS, PEREIRA TS. O agrotóxico nosso de cada dia. Vittalle. Revista de Ciências da Saúde, 2016;28;14-26. 27. SANTOS MBG, et al. Riscos ocupacionais no processo de fabricação de aguardente de cana de açúcar. Anais... XXXVI ENCONTRO NACIONAL DE ENGENHARIA DE PRODUCÃO Contribuições da Engenharia de Produção para Melhores Práticas de Gestão e Modernização do Brasil João Pessoa/PB, Brasil, de 03 a 06 de outubro de 2016.

28. SENAR-AR/PE. Atualização 2013. Alterações introduzidas pela IN/RFB № 971, de 13 de novembro 2009. Disponível em: $\quad<$ https://docente.ifsc.edu.br/lucia.martins/MaterialDidatico/Agroneg\%C3\%B3cio/AGROINDUSTRIAreceita\%20federal.pdf>. Acesso em: 28 ago. 2019.

29. SHENG K, et al. Effect of ammonia and nitrate on biogas production from food waste via anaerobic digestion. Biosyst. Eng., 2013;116:205-212.

30. SILVA TL, ALMEIDA VC. Influência do calor sobre a saúde e desempenho dos trabalhadores. Anais... SIMPÓSIO MARIGAENSE DE ENGENHARIA DE PRODUÇÃO; 2010.2 Disponível em: <www.dep.uem.br/gdct/index.php/simeprod/article/download/616/622>. Acesso em: 15 ago 2019.

31. SOARES R. As Diversas Formas de Conservação de Alimentos. São Paulo: Hôtelier News; 2010:1-5.

32. SOUSA MNAS, ASSIS EV, FEITOZA ANA (Orgs.). Saúde do trabalhador: abordagem em múltiplos contextos. Curitiba: Editora CRV; 2014.

33. SOUSA MNA, MEDEIROS AC, MARACAJÁ PB (Orgs.). Tecnologia, desenvolvimento e meio ambiente em sistemas agroindustriais. Curitiba: CRV; 2018.

34. TEVES MLU. Ácido Sulfúrico. Rev. abril, 2003. Disponível em: <https://www.oswaldocruz.br/download/fichas/\%C3\%81cido\%20sulf\%C3\%BArico2003.pdf >. Acesso em: 15 ago 2019. 35. VIANA LG, CRUZ PS. Reaproveitamanto de resíduos agroindustriais. Anais... IV CONGRESSO BAIANO DE ENGENHARIA SANITÁRIA E AMBIENTAL, Cruz das Almas, Bahia - 13 a 16 de julho de 2016. Disponível em: <http://cobesa.com.br/2016/download/cobesa-2016/IVCOBESA-133.pdf>. Acesso em: 20 ago 2019. 
36. VIEIRA FILHO JER, VIEIRA ACP. Inovação na agricultura brasileira: uma reflexão a partir da análise dos certificados de proteção de cultivares. Texto para Discussão (IPEA. Brasília), 2013;1866:1-34.

37. WILKINSON J (Coord.). Perspectiva do investimento em agroindústria. Rio de Janeiro: UFRJ; 2009.

38. WOOD RND. Intoxicação por Monóxido de Carbono. 2018. Disponível em: <https://www.hospitalinfantilsabara.org.br/sintomas-doencas-tratamentos/intoxicacao-por-monoxido-de-carbono/>. Acesso em 12 ago 2019.

39. ZANI MLC, LAZZARINI R, SILVA-JUNIOR JS. Pés de imersão em água morna entre trabalhadores de lavagem de automóveis. Rev Bras Med Trab. 2017;15(3):217-21.

40. ZAVALA P, MACARIO G, VICHI FF. El sector citrícola de Nuevo León: caracterización del sistema agroalimentario como plataforma de integración del productor con la agroindustria. Región y sociedad,2018;30(71):1-47. 